\title{
Facing Cell Autophagy in Gastric Cancer - What Do We Know so Far?
}

\author{
Ting Xiu ${ }^{1,2}$ \\ Qie Guo' \\ Fan-Bo Jing' \\ 'Department of Clinical Pharmacy, The \\ Affiliated Hospital of Qingdao University, \\ Qingdao, Shandong, 266003, People's \\ Republic of China; ${ }^{2}$ Department of \\ Pharmacology, School of Pharmacy, \\ Qingdao University, Qingdao, 26602I, \\ People's Republic of China
}

\begin{abstract}
Autophagy is a process by which misfolded proteins and damaged organelles in the lysosomes of tumor cells were degraded reusing decomposed substances and avoiding accumulation of large amounts of harmful substances. Here, the role of autophagy in the development of malignant transformation of gastric tumors, and the underlying mechanisms involved in autophagy formation, and the application of targeted autophagy in the treatment of gastric cancer were summarized.
\end{abstract}

Keywords: autophagy, gastric cancer, autophagy-related genes, lysosomes, targeted therapy

\section{Introduction}

Gastric cancer (GC) is the second leading cause of cancer-related mortality worldwide, with high morbidity and high-grade malignancy. ${ }^{1}$ Although surgical removal is a curative treatment, systemic chemotherapy is an optimal therapeutic strategy for GC patients who are diagnosed at an advanced stage and consequently have distant metastases and poor prognosis, offering better outcomes than surgery alone. ${ }^{2}$ In particular, chemotherapy has been approved as a treatment option for gastrointestinal cancers. ${ }^{3}$ Unfortunately, in GC patients with chemotherapeutic drugs, tumor metastasis and local recurrence become increasingly common due to chemoresistance. ${ }^{4}$ Autophagy is a homeostatic process in which the aged and damaged proteins or certain organelles of the cell are encapsulated into vesicles, and were bound with lysosomes to form autophagic lysosomes, and finally were degraded as the contents encapsulated by autophagy-related genes (ATGs) under starvation, hypoxia or other specific cellular stress conditions. ${ }^{5}$ If autophagy is over-activated and exists at high levels for a long time, it may damage the cells themselves and cause autophagic death of the cells. Therefore, autophagy has dual effects of promoting cell survival and inhibiting cell survival. ${ }^{6}$ In addition, autophagy participates in regulating the expression of many oncogenes and tumor suppressor genes, thus promoting and inhibiting the occurrence and development of tumors. ${ }^{7,8}$

Herein, we view the putative role of autophagy exerted in GC development and lists some of the common autophagy markers in recent years and the prognostic markers related to autophagy in the diagnosis and treatment of $\mathrm{GC}$, and also summarizes the regulatory mechanism of autophagy in GC and the role of autophagy in promoting and inhibiting tumors and drug resistance. Finally, we discuss autophagy as an important treatment target for GC.
Correspondence: Fan-Bo Jing

Email jingbfl78@sina.com 


\section{Types of Autophagy}

Autophagy is divided into macroautophagy, microautophagy and chaperone-mediated autophagy.

1) Macroautophagy represented the process that the formation of autophagosomes encapsulating degradable substances by membranes within cells, which are then transported to and fused with lysosomes to form autophagolysosomes and degrade their internal components; 2) Microautophagy represented the process that the degraded substances are directly encapsulated and degraded by their own lysosomal membranes; 3) Chaperone-mediated autophagy (CMA) indicated that degraded soluble proteins bind chaperone proteins to form complexes that are transported into lysosomes for degradation. ${ }^{9}$ The most prominent feature of macroautophagy is the formation of bilayermembrane autophagosomes, which are the most common and clinically significant types of autophagy.

\section{Differentially Expressed Autophagy Markers in GC Cells}

Differential expression of ATGs in GC cells which presented an indicator of autophagy formation is firstly discussed.

\section{LC3}

Microtubule-associated protein 1 light chain 3 (MAPLC3), also known as the LC3, is the first discovered autophagosome marker protein, including three subtypes of LC3A, LC3B and LC3C that were called as LC3-I and LC3-II. When autophagy occurs, LC3-I is ubiquitinated and bound to phosphatidylethanolamine on the surface of the autophagic membrane to form type II LC 3. ${ }^{10}$ Therefore, autophagy can be identified according to the level of type II LC3 expression.

\section{P62}

P62, also known as sequestosome 1 (SQSTM1), has multiple domains, including PB1 domain, TB domain, a keap1 interaction region (KIR), a ubiquitin-related region, and so on. Several studies have shown that the level of p62 is negatively correlated with the level of autophagy in GC. ${ }^{11,12}$

\section{Beclin-I}

Beclin-1, a yeast ATG6 homolog, is the first step in autophagosome formation and one of the important initiators of autophagy, and the expression level of Beclin-1 tends to rise during autophagy. ${ }^{13}$ Beclin-1 is necessary to initiate the process of autophagy, decreased levels of autophagy in $\mathrm{GC}$ can be revealed by decreased expression of Beclin- $1 .{ }^{14}$ In addition, Beclin-1 plays a key role in promoting autophagy-induced apoptosis resistance, and there is a positive correlation between the expression of Beclin-1, Bcl-2 and Bcl-xl, Beclin-1 can also induce the Bcl-2 and Bcl-xl expression, alternatively, down-regulate the protein levels of Bak and Bax, Cyto-C, Apaf-1, Smac, Caspase and cleaved Caspase, and thus inhibiting the apoptosis of GC cells but inducing autophagy in GC cells. ${ }^{15,16}$

\section{Prognostic Value of Related Proteins in GC PCDHGA9}

Protocadherin gamma subfamily a, 9 (PCDHGA9) is a member of the cadherin superfamily. PCDHGA9 can inhibit tumor proliferation by inducing GC cell autophagy, and its reduced expression may serve as an independent prognostic marker in GC. ${ }^{17}$

\section{ATG5}

Autophagy is a highly programming dynamic process, mainly executed by the autophagy-related gene family, and regulated by key kinases such as mTOR, PI3k/AKT, AMPK, MAPK. ${ }^{18,19}$ ATG5 participates in autophagosome elongation as a central regulator, and is necessary for autophagy, which is associated with chemotherapy resistance of cancer cells. ${ }^{20}$ MRP1 (multidrug resistancerelated protein-1) is an $\mathrm{ABC}$ transmembrane transporter that promotes the MDR phenotype in GC, and both ATG5 and MRP1 are highly expressed in GC. ATG5 expression is positively correlated with MRP1, and high expression of ATG5 may lead to more aggressive and malignant phenotype of GC to some extent, which may provide valuable information for better evaluation of chemotherapy effect in GC patients. The expression of ATG5 and MRP1 can be used as independent prognostic indicators to predict overall survival (OS) and disease-free survival (DFS) of GC patients. $^{21}$

\section{ASSI}

Arginine succinate synthase 1 (ASS1) is the rate-limiting enzyme in arginine biosynthesis. ${ }^{22}$ Emerging evidence has shown that loss of ASS1 expression is associated with tumor aggressiveness and worse prognosis in many tumor types, including myxofibrosarcoma, ${ }^{23}$ bladder 
cancer $^{24}$ and osteosarcoma, ${ }^{25}$ However, other studies have suggested that ASS1 is highly expressed in GC tissues. Ectopic expression of ASS1 protects GC cells from apoptosis induced by chemotherapeutic drugs by activating AKT and mTOR, thereby inhibiting the autophagy process and enhancing the invasive potential through the accumulation of active $\beta$-catenin, Snail and Twist. ASS1 may be a useful prognostic marker for predicting survival and metastasis of GC patients. ${ }^{26}$

\section{SPI}

SP1 belongs to the SP1 multigene family (including SP2, SP3 and SP4) and plays an important role in the occurrence and development of tumors. ${ }^{27}$ The expression of SP1 in GC tissues was significantly increased, and it was closely related to the survival rate of patients. ${ }^{28}$ It plays a negative role in regulating autophagy by directly binding to the promoter of p62 and increasing the expression level of p62. Since the SP1-p62 axis may promote the occurrence of $\mathrm{GC}$, it may be a prognostic marker for the diagnosis of GC. ${ }^{29}$

\section{Autophagy Regulation Mechanism in GC Cells}

\section{Regulation of Autophagy by microRNAs (miRNAs)}

MiRNAs are a class of endogenous small non-coding RNA molecules with a length of about 19-22 nucleotides that regulate gene expression by binding to the 3'-UTR of mRNAs. ${ }^{30}$ Studies in the past few years have found that the dysregulation of miRNAs is associated with the biological activity of $\mathrm{GC}^{31}$ and may serve as a diagnostic or prognostic marker for GC cells. With the involvement of miRNAs, the complex autophagy-mediated regulatory mechanisms become more complex during gastric carcinogenesis $^{32}$ and needed to be further explored.

\section{Regulation of Autophagy by miRNAs as Tumor Suppressor Genes}

The study showed that the expression of miR-1265 in GC specimens is lower than that in adjacent normal tissue specimens. The target gene of miR-1265 is calcium-binding protein 39 (CAB39), which plays a key role in the LKB1STRAD-CAB39 complex, $^{33,34}$ at the Thr172 junction of LKB1 with STRAD and CAB39, the phosphorylation of AMPK increased by more than 100 times, ${ }^{35,36}$ autophagy induced by the CAB39-LKB1-AMPK axis is carcinogenic in
GC cells; therefore, miR-1265 inhibits GC progression and autophagy by decreasing the expression of CAB39 and regulating the AMPK-mTOR signaling pathway. ${ }^{37}$ MiR-495-3p reverses MDR by inhibiting autophagy through regulating mTOR signaling, and down-regulation of miR-495-3p is associated with the malignant phenotype of GC patients. ${ }^{38}$ Other miRNAs, including miR-375, ${ }^{39}$ miR-21, ${ }^{40}$ miR-361$5 \mathrm{p},{ }^{41}$ which act as autophagy inhibitors, regulate autophagy and reduce $\mathrm{GC}$ activity by regulating $\mathrm{mTOR}$ signaling. In addition, the expression of miR-181a, ${ }^{42}$ miR-30a, ${ }^{43}$ miR-let $-7 \mathrm{a},{ }^{44}$ miR-133a-3p, ${ }^{45}$ miR-532-3p ${ }^{46}$ and so on have been confirmed to be associated with the decreased proliferation ability of GC cells.

\section{Regulation of Autophagy by miRNAs as Oncogenes} It has been showed that miR-21 inhibits autophagy through the $\mathrm{PI} 3 \mathrm{~K} / \mathrm{AKT} / \mathrm{mTOR}$ pathway and is associated with DDP resistance in GC cells. ${ }^{40}$ In addition, ultraviolet radiation resistance-associated gene(UVRAG) may interact with other Bcl-2 family members to induce autophagy and trigger GC death, ${ }^{47}$ and miR-183 inhibits starvationinduced autophagy and apoptosis in human GC cells by targeting the 3'-UTR region of UVRAG; therefore, miR183 is regarded as an autophagy-related oncogene. ${ }^{48}$ However, it is contradictory that miR-183 may play a role as a GC suppressor. ${ }^{49}$ Besides that, miR-20a as an oncogene is highly expressed in GC which is expected to be a biomarker for clinical detection. ${ }^{50}$

Therefore, exploring the new function of miRNAs in the regulation of autophagy, which can help us develop more effective treatment strategies and provide new therapeutic targets for the study of resistance mechanisms in GC, thereby improving the clinical efficacy of cancer patients.

\section{Regulation of Autophagy by Long Non-Coding RNAs (IncRNAs)}

New evidence shows that lncRNAs-HAGLROS interact with $\mathrm{mTORC} 1$ and activate mTORC1 signaling pathway to inhibit autophagy, thereby promoting GC cell overproliferation and maintaining its malignant phenotype, high expression of HAGLROS contributes to the development and poor prognosis of GC. ${ }^{51}$ Numerous studies have shown that IncRNAs contribute to chemoresistance in multiple cancers, ${ }^{52-54}$ and have been proven to be the effective prognostic indicators in multiple cancers. ${ }^{55-57}$ MALAT1, as a competitive endogenous RNA of miR$23 \mathrm{~b}-3 \mathrm{p}$, has been demonstrated to attenuate the inhibitory 
effect of miR-23b-3p on ATG12, and increase the expression of ATG12, leading to chemotherapy-induced GC cell autophagy and drug resistance. ${ }^{58}$ More interestingly, ARHGAP5-AS1 is a new drug-resistant lncRNA, which is up-regulated in GC resistant cells and can reverse chemoresistance after being knocked out. ${ }^{59}$

\section{Regulation of Autophagy by PI3K/AKT/ mTOR Signaling Pathway}

As reportedly, autophagy is regulated by a variety of signaling pathways, among which the PI3K/AKT/mTOR pathway is well-recognized. The PI3K/AKT/mTOR pathway is composed of three acting molecules, PI3 kinase (PI3K), protein kinase $\mathrm{B}(\mathrm{PKB} / \mathrm{AKT})$ and mammalian rapamycin target protein (mTOR) ${ }^{60}$ It is a central regulatory mechanism and promotes the growth and proliferation of tumor cells and inhibits autophagy at the same time. ${ }^{61}$ Aberrantly activated PI3K/AKT/mTOR pathway in GC plays an important role in tumor aggressive growth, prolonging tumor cell survival time and generating chemoresistance. $^{62}$ A large number of studies have shown that AKT activation can increase the resistance of GC cells to various chemotherapeutic drugs such as 5-fluorouracil (5-FU), doxorubicin, mitomycin $\mathrm{C}$ and cisplatin (CDDP), ${ }^{63}$ in addition, it can also activate downstream mTOR molecules, which are responsible for regulating cell growth, proliferation and protein synthesis, mTOR pathway negatively regulates the process of autophagy and has an antagonistic effect on autophagy, so mTOR inactivation is a key step to activate autophagy. ${ }^{64}$ For example, the above-mentioned flavonoids can play an anti-cancer role by down-regulating the PI3K/AKT/mTOR pathway, thereby leading to G2/M cell cycle arrest, autophagy and apoptosis of GC cells. ${ }^{65}$ In addition, it has been mentioned that silencing YWHAZ can also induce apoptosis and autophagy of GC cells by inhibiting the activation of PI3K/AKT/mTOR signaling pathway in BGC-823 cells. $^{66}$

\section{Regulation of Autophagy by AMPK Signaling Pathway}

AMPK signaling pathway can jointly regulate cell biological behaviors such as cell proliferation, growth, apoptosis, autophagy and play an anti-cancer role by interacting with PI3K-AKT signaling pathway, MAPK signaling pathway and a variety of transcription factors. Clinicopathological data showed that AMPK activity in tumor tissues is lower than that in normal tissues and inadequate AMPK activity is considered to be one of the reasons for the occurrence and development of malignant tumors. ${ }^{67,68}$ Recent studies have revealed that Perillaldehyde can activate AMPK by phosphorylation of LKB1 at sites S307 and S428, and AMPK directly stimulates autophagy of GC cells through phosphorylation and activation of ULK1 and inhibits the growth of GC cells. ${ }^{69}$ In addition, Metadherin (MTDH) plays an important role in 5-FU, doxorubicin, CDDP, etoposide and paclitaxel resistance, particularly, MTDH regulates ATG5 expression by inducing AMPK phosphorylation, suggesting that MTDH may activate autophagy through the AMPK/ATG5 signaling pathway and promote drug resistance in $\mathrm{GC}$ cells. $^{70}$

\section{Regulation of Autophagy by Helicobacter pylori}

Helicobacter pylori is a Gram-negative bacterium parasiticed in human gastric mucosa, which is closely related to the occurrence and development of $\mathrm{GC}^{71}$ Helicobacter pylori can produce a variety of pathogenic factors, such as urease, vacuolating toxin (VacA), cytotoxin-related gene A (CagA) and lipopolysaccharide, etc., these toxic factors are a putative force in triggering the occurrence and development of cancer. ${ }^{72}$ In particular, VacA is a necessary and sufficient factor to produce autophagy in gastric epithelial cells, ${ }^{73,74}$ while CagA may inhibit autophagy through the c-Met-PI3k/AKTmTOR signaling pathway. Some scholars have also shown that Helicobacter pylori can lead to the upregulation of HO-1 expression by inducing Nrf2 activation through the production of ROS. CO is a by-product of HO-1, which can directly induce autophagy of GC cells while inhibiting apoptosis, and protect the body from toxin damage. ${ }^{75}$ At present, the research on the molecular mechanism of autophagy regulation by Helicobacter pylori is still in progress.

\section{Duality of Autophagy in GC Inhibitory Effect of Autophagy on GC Growth}

Autophagy is considered to be a favorable mechanism to inhibit tumor formation at multiple stages, thus preserving genome stability, eliminating endogenous sources of ROS, and maintaining bioenergetic functions. 
In the early stage of tumorigenesis, autophagy can maintain cell homeostasis to inhibit the occurrence and development of GC. In fact, autophagic cell death is different from apoptosis. In GC cells, autophagy and apoptosis exist simultaneously, and the interaction between them regulates cell death relatively independently. Apoptosis exists downstream of autophagy, and autophagy is a necessary condition for apoptosis. ${ }^{76}$ For example, both autophagy and apoptosis can be regulated by the PI3K AKT/mTOR signaling pathway, which can coordinately regulate the fate of GC cells. ${ }^{77}$ Methylxanthine derivatives such as caffeine and theophylline have been showed to inhibit the PI3K/Akt/mTOR pathway by activating PTEN, thereby effectively inducing apoptosis and autophagy of GC cells and inhibiting the proliferation of GC cells. ${ }^{78}$ ER stress and its UPR can also be related to the survival, development and drug resistance of GC cells through various cellular processes such as autophagy. Melatonin promotes cell autophagy through ER stress, thereby promoting apoptosis of GC cells and inhibiting their growth, proliferation and invasion. ${ }^{79,80}$ Tetrandrine can induce mitochondrial apoptosis and inhibit Akt/mTOR pathway, so it can induce autophagy and apoptosis of HGC-27 cells, thereby exerting anti-tumor activity and leading to gastric tumor cell death. Therefore, in the process of tetrandrineinduced anti-tumor, autophagy and apoptosis synergistically promote tumor cell death. ${ }^{81}$

\section{Promoting Effect of Autophagy on GC Growth}

As a stress response mechanism of cells to internal and external stimuli, autophagy can not only promote cell death but also prolong the survival time of cancer cells exposed to chemotherapeutic drugs. Autophagy can protect tumor cells from damage by exercising normal autophagy process. $^{82}$ Otherwise, autophagy segregates organelles such as mitochondria within cells, which can effectively prevent the proliferation of pro-apoptotic factors within cells and thus help tumor cells escape the threat of apoptosis. Inhibition of autophagy in cancer cells can improve the toxicity of anti-tumor drugs and reverse drug resistance. ${ }^{83,84}$ Both apatinib and astragalus polysaccharides can induce autophagy and apoptosis but inhibit the metastasis and invasion of GC, While autophagy inhibitors also can significantly enhance the apoptosis of AGS cells, so it seems that the elevated autophagy induced by apatinib protects cells from apoptosis.
Elevated autophagy may have adverse effects on chemotherapy, so autophagy inhibition can sensitize resistant GC cells and enhance the anti-tumor effect of chemotherapeutic drugs ${ }^{85}$ Increasing evidence shows that autophagy inhibition has a pro-apoptotic effect on human GC cells. For example, cinobufacin can induce the production of ROS, which triggers apoptosis and autophagic cell death by activating the ROS/JNK/p38 axis. When autophagy is inhibited, increased pro-apoptotic protein expression, disordered mitochondrial membrane potential, and increased ROS production are found, so inhibition of autophagy enhances cinobufagin-induced apoptosis, which may occur in part through the mitochondrial programmed cell death pathway. ${ }^{86}$

\section{Targeted Autophagy in the Treatment of GC}

Although the relationship between autophagy and cancer is still controversial, the involvement of common regulatory pathways makes autophagy a promising target in cancer therapy. Tumor cells to chemotherapy/radiotherapy can be sensitized by inhibiting the cytoprotective effect of autophagy, thereby inducing autophagic death of antiapoptotic cells.

\section{Inhibitors of Autophagy}

When exposed to malignant environments, the occurrence or evasion of apoptosis usually depends on the activation of rescue mechanisms such as autophagy. ${ }^{87}$ Autophagy, as a mechanism to promote resistance to chemotherapy or radiotherapy, impairs the efficacy of anti-cancer treatment strategies. If autophagy of cancer cells is inhibited, it will lead to biological effects such as impaired mitochondrial metabolism, redox imbalance, nucleotide consumption, reduced energy supply and so on. Therefore, inhibition of autophagy can be used as a tool to enhance chemosensitivity. Common autophagy inhibitors can be divided into the following categories:

\section{Inhibitors of Autophagosome Formation}

Class III PI3K inhibitor, 3-methyladenine (3-MA), inhibits autophagy by blocking the initiation of autophagy and preventing the formation and development of autophagosomes, thereby significantly reducing the expression of Beclin- $1,{ }^{88}$ which has been proved to have anti-cancer effects or improve the efficacy of anti-tumor therapy. 


\section{Inhibitors of Autophagosomal Lysosomal Fusion}

Chloroquine (CQ) is an antimalarial drug. Recently, the exact mechanism of blocking autophagy by chloroquine derivatives has been confirmed. ${ }^{89}$ It inhibits autophagy mainly by disrupting the fusion of autophagosome and lysosome without direct effect on the acidity of organelles. ${ }^{90}$ It has been proved to have anti-cancer effect or improve the efficacy of anti-tumor therapy. The combination of CQ and 5-FU can further inhibit the proliferation of GC stem cells. ${ }^{91}$ In addition, BAF-1 can also block autophagy by preventing the fusion between lysosome and autophagy, resulting in the decrease of LC3-II level and the enhancement of p62. ${ }^{92}$ However, the relationship between BAF-1 and the treatment of GC has not been reported.

\section{Gene Silence of ATGs at the Transcriptional Level} Using siRNA or miRNA-mediated silencing strategies, the knockout of autophagy-related genes subsequently inhibits autophagic activity. Sequence-specific DNA or RNA analogues block the expression of gene sequences with high specificity, providing the possibility to design customized molecules with potential anti-cancer effects at relatively low cost. In this sense, in addition to oncogenes, some important regulators of autophagy mechanisms, such as ATG3, ATG4B, ATG4C, ATG5, Beclin-1, ATG10 and ATG12, can also be targeted. ${ }^{93}$ It has been found that increased ATG5 expression was significantly associated with OS and DFS of GC patients and ATG5 was overexpressed in drug-resistant GC cell lines, silencing ATG5 (siRNA-ATG5-695) can sensitize resistant cells to chemotherapy again. ${ }^{94}$ In addition, inhibition of ATG5 expression by siRNA inhibits autophagy can enhance the apoptosis of GC cells activated by cinobufacin through mediating ROS production and mitochondrial programmed cell death pathway.

Chronic stress, especially adrenergic signaling, is involved in the occurrence and development of various cancers. Norepinephrine-induced autophagy activation in GC cells is an important mechanism for the development of GC. ${ }^{95,96} \beta 2$-adrenergic receptor (ADRB2) is a specific $\beta$ adrenergic receptor subtype that causes catecholamine release. Knockout of the ADRB2 gene may decrease the expression of autophagy-related genes including Beclin-1 and inactivate the AMPK-ULK1 pathway, thereby attenuating autophagy and inhibiting the growth of human gastric cells. ${ }^{97}$
Rab5a, a member of the Rab family, is not only involved in intracellular material transport and protein classification but also in the process of autophagy. Rab5a, an upstream regulator of mTOR, can inhibit autophagy and promote drug resistance in GC cells by activating mTOR. Therefore, knockout of Rab5a can inhibit mTOR activity, increase autophagy of SGC7901 cells and reverse their drug resistance. ${ }^{98}$

\section{AMPK Inhibitors}

Previous studies have found that AMP-activated protein kinase $\alpha$ (AMPK $\alpha$ ) activates autophagy by activating ULK1 and inhibiting mTOR/p70S6K signaling. ${ }^{99}$ Norepinephrine, a stress hormone, promotes autophagic flux through AMPK-ULK1 pathway, therefore activating tumor-promoting autophagy and accelerating GC progression. $^{100}$ And Danggui-Sayuk-Ga-OsuyuSaenggang-Tang (DSGOST) induces autophagy by activating AMPK/ULK1 signaling, thereby activating EMT and exosomes to promote chemoresistance, so survivalpromoting autophagy is one of the mechanisms of GC resistance to DSGOST. By using compound C, a wellknown inhibitor of AMPK, to block DSGOST-mediated autophagy and reduce drug resistance, thereby promoting the death of GC cells. ${ }^{101}$ Therefore, AMPK inhibitors may effectively improve the efficacy of anti-cancer drugs.

\section{NSAIDs}

Gastrointestinal epithelial cells are the target cells of nonsteroidal anti-inflammatory drugs (NSAIDs). When used to treat pain and inflammation, these drugs have harmful effects on digestive epithelial cells, which are their main side effects. ${ }^{102}$ However, this activity of NSAIDs has a linked positive effect by inhibiting tumorigenesis in gastrointestinal tissues and is considered as an adjunct drug to chemotherapy. It has been shown that the decrease of lysosomal acidity and the increase of membrane permeability in indomethacin-treated AGS cells interfere with the normal function of lysosomes and the decrease of cathepsin activity in cells, thus insufficient degradation of autophagic substrates destroys the autophagic flux, thereby increasing the sensitivity of GC cells to cytotoxic drugs. ${ }^{103}$

\section{Cancer Stem Cell (CSC) Marker Inhibitors}

Studies have shown that a series of CSC markers, such as CD133, CD44, CD24, ALDH, CDCP1, EpCAM and LGR5, play a key role in tumorigenesis. ${ }^{104}$ For example, CD133, a 5-transmembrane glycoprotein, is first identified as a marker of hematopoietic stem cells and plays a key 
role in a variety of cancers. ${ }^{105}$ It has been demonstrated that $\mathrm{CD} 133$ promotes CDDP resistance of gastric cancer stem cells (GCSCs) by activating the PI3K/AKT/mTOR signaling pathway to increase the proliferation, antiapoptotic and autophagic abilities of GC cells. Therefore, the IC50 value of GC cells transfected with sh-CD133 is decreased by regulating PI3K/AKT/mTOR signaling pathway to reduce autophagy, promote Bax expression and enhance the sensitivity of Cis-KATO-III cells to CDDP. ${ }^{106}$

\section{Activators of Autophagy Pectolinarigenin (PEC)}

PEC is a natural flavonoid, widely found in Cirsium chanroenicum and some species of Citrus plant, with antiinflammatory, anti-cancer and other pharmacological effects. ${ }^{107,108}$ The PI3K/AKT/mTOR signaling pathway is activated in a large amount of cancers including $\mathrm{GC}$ to regulate cell growth and stimulate cell survival. Previous studies have also shown that PEC treatment induces an acute decrease in the phosphorylation of the mTOR targets p70 ribosomal protein $\mathrm{S} 6$ kinase (p-p70S6K), 4E-BP1 and p-eIF4E. ${ }^{109}$ Therefore, PEC may be a new anti-tumor drug to improve the therapeutic effect of chemotherapeutic drugs towards human GC, and the combined treatment of PEC and GC can be further studied.

\section{Kaempferol}

Kaempferol is a natural flavonoid widely found in many fruits, vegetables and traditional herbs. ${ }^{110}$ Studies have shown that kaempferol can mediate the activation of IRE1JNK-CHOP pathway, as reportedly, kaempferol can induce autophagic cell death in GC cells by disrupting the Bcl2-beclin-1 complex, in which the phosphorylation of Bcl-2 was induced by the activation of JNK1. On the other hand, kaempferol induces LC3B expression and activates autophagic cell death by inhibiting G9a-mediated epigenetic changes. ${ }^{111}$ In another study, kaempferol can also inhibit the proliferation of human GC SNU-216 cells by upregulating miR-181a and inactivating the MAPK/ERK and PI3K pathways to induce cell autophagy without effect on apoptosis. ${ }^{112}$

\section{Curcumin}

Curcumin is derived from Curcuma longa, in recent years, there have been many studies on the anti-cancer effect of curcumin. Studies have found that curcumin has the effects of inducing apoptosis, inhibiting angiogenesis and improving the sensitivity of chemotherapy and radiotherapy. ${ }^{113}$
There is considerable evidence that curcumin has a variety of molecular targets, including STAT3, ${ }^{114}$ reactive oxygen species (ROS) ${ }^{115} \mathrm{NF}-\mathrm{\kappa B}^{116}$ and cyclooxygenase-2. ${ }^{117}$ In addition, recent studies have found that curcumin can activate the P53 signaling pathway by up-regulating P53 and P21 and down-regulation of PI3K, p-AKT and p-mTOR to inhibit the PI3K pathway, thereby inhibiting the proliferation of GC cells and inducing GC cell autophagy and apoptosis. ${ }^{118}$

\section{Magnoline (Mag)}

Mag is an alkaloid isolated from Coptis chinensis, which has many biological properties such as antifungal, inhibition of $\alpha$-tyrosinase, anti-inflammatory and anti-tumor. ${ }^{119}$ Studies have shown that Mag-treated GC cells can induce the production of a large number of ROS, and ROS, as an upstream factor regulating JNK and AKT signaling pathways, can lead to JNK phosphorylation, MAPK family proteins have been reported to mediate AKT signaling, ${ }^{120}$ therefore, JNK can not only mediate $\mathrm{S} / \mathrm{G} 2$ phase cycle arrest and apoptosis of GC cells but also inhibit the activation of AKT, thereby inhibiting the AKT/mTOR pathway to induce autophagy in GC cells. ${ }^{121}$ These findings provide strong evidence for Mag to become a new candidate for the treatment of GC.

\section{Melatonin}

Melatonin is an indoleamine synthesized by the pineal gland, retina, brain, heart and gastrointestinal system, ${ }^{122}$ which can inhibit the proliferation of a variety of human tumors, including GC. ${ }^{123}$ Studies have shown that melatonin can induce endoplasmic reticulum stress and its unfolded protein response (UPR). Glucose regulated protein 78 (GRP78) is an important endoplasmic reticulum (ER) molecular chaperone, which normally binds to Inositol-requiring transmembrane kinase/endonuclease $1 \alpha$ (IRE1 $\alpha$ ) under normal physiological conditions, ${ }^{124}$ while IRE $1 \alpha$ is released under ER stress, IRE1 $\alpha$ can recruit tumor necrosis factor receptor related factor 2 (TRAF2) at the ER,activate apoptosis signal-regulating kinase 1 (ASK1) and then phosphorylate JNK, the activated JNK can stimulate Bcl-2 to release Beclin-1, thus activating autophagy and promoting the development of GC cell death. ${ }^{125,126}$ Considering the role of melatonin in the progression of GC cells, indoleamine may be a strong candidate for anti-tumor therapy.

\section{Ginsenoside}

Ginsenoside, the main component of ginseng, has been used as a candidate drug for the treatment of a variety of 
cancers and can inhibit the proliferation of tumor cells and tumor growth. ${ }^{127}$ Studies have shown that ginsenosides Rg3 and F2 can induce apoptosis of GC cells. ${ }^{128,129}$ Recently, new studies have found that ginsenoside $\mathrm{Rg} 5$ can inhibit the proliferation of GC in vivo and in vitro with little side effects, $\mathrm{Rg} 5$ can damage the mitochondrial function of GC cells and induce the production of ROS significantly increased, overproduction of ROS can activate the MAPK pathway to participate in $\mathrm{Rg} 5$-induced $\mathrm{G} 2 / \mathrm{M}$ phase arrest, apoptosis and autophagy of GC cells, Rg5 can simultaneously induce autophagy and apoptosis in $\mathrm{GC}$ cells, and the interaction between them promotes each other. ${ }^{130}$ Ginsenoside has anti-cancer effects and various anti-cancer molecular mechanisms, which will be a new and promising drug for clinical targeted treatment of GC.

\section{Methylxanthine Derivatives}

Methylxanthine derivatives are extracted from plants, including caffeine and theophylline, in addition to exert its effects on the nervous system, methylxanthine derivatives can also reduce the risk of certain cancers, such as GC. ${ }^{131}$ In particular, the above-mentioned caffeine can promote PTEN expression and negatively regulate PI3K/ AKT signaling pathway, thus promoting cell proliferation, apoptosis and migration, and also enhancing autophagy and apoptosis of GC cells, ${ }^{78}$ in addition, caffeine and theophylline have been shown to induce apoptosis of cancer cells through Bcl-2 family proteins. ${ }^{132}$ Since the Bcl2-Beclin-1 complex is involved in regulating the interrelationship between apoptosis and autophagy signaling pathways, ${ }^{133}$ we hypothesize that methylxanthine derivatives could induce apoptosis-related autophagy in GC cells. Through a step-by-step study of its anti-cancer effects, methylxanthine derivatives may show potential as anti-cancer therapies.

\section{Sophocarpine}

Sophocarpine is an important alkaloid extracted from the traditional Chinese medicine Sophora flavescens, which was regarded as a anti-virus, anti-tumor, and antiinflammation drug, ${ }^{134}$ and has been applied in the treatment of GC. Sophocarpine can induce autophagy in human GC cells by regulating the PTEN/PI3K/AKT pathway, ${ }^{135}$ thereby inducing apoptosis and inhibiting proliferation of GC cells, it also shows anti-tumor properties both in vitro and vivo. These findings may provide a new approach for the development of GC treatment.

\section{Conclusions and Prospects}

The role of cell autophagy in tumor development is complex, and autophagy can produce different effects in different tumor types and tumor stages. Therefore, clarifying the role of autophagy not only has theoretical significance but also has very important clinical application value. There is evidence that autophagy may play a role in tumor inhibition in the early stage of malignant transformation and/or cancer progression, while in the late stage, autophagy shows considerable protocarcinogenicity to promote tumor maintenance and resistance to chemotherapy. The effect of anti-tumor therapy based on autophagy regulation depends on the actual level of intracellular autophagy, which is influenced by many factors. Among them, PI3K and mTOR signaling pathways have been confirmed as the main signaling pathways regulating autophagy, other autophagy-related pathways (p53, MAPK or PTEN) need to be studied in the future. In addition, the important role of miRNA and Helicobacter pylori in the regulation of autophagy is gradually being paid attention to. Although autophagy activator and inhibitor in the treatment of GC have achieved significant clinical trial results, it has broad clinical application prospects. However, the two opposite roles of autophagy in the treatment of GC also need to be considered. On the other hand, many studies have confirmed that autophagy and apoptosis can coexist or occur sequentially, ${ }^{136,137}$ and there can be some interaction between them. In particular, new evidence have summarized the complex relationship between autophagy and chemotherapy resistance in GC because of the diversity and unknown mechanism of antitumor drug resistance, which leads to ineffective treatment and poor prognosis in patients with advanced GC. However, the early diagnosis and screening of GC is a crucial step in the whole treatment process. Therefore, the related proteins and autophagy-related genes involved in the process of autophagy are expected to become new targets and prognostic indicators for molecular targeted therapy of GC. This paper also summarizes and discusses the differentially expressed proteins and genes in GC. Further understanding of autophagy markers can provide a new choice for prognostic indicators and therapeutic targets of GC. In addition, more evidence shows that many natural products can trigger chemotherapy resistance by regulating different signaling pathways. Therefore, natural products alone or in combination with autophagy modulators and/or chemotherapeutic drugs may have 
a good effect on drug-resistant cancer. However, further studies are needed to identify molecular mechanisms and specific targets and to authenticate the effectiveness and safety of these strategies in clinically relevant cancer models. How autophagy activity is regulated differently in GC, or which factors determine the tissue-specific inhibition and/or activation of autophagy should be corroborated.

\section{Disclosure}

The authors report no conflicts of interest in this work.

\section{References}

1. Fock KM. The epidemiology and prevention of gastric cancer. Aliment Pharmacol Ther. 2014;40(3):250-260. doi:10.1111/ apt. 12814

2. Yoon H, Kim N. Diagnosis and management of high risk group for gastric cancer. Gut Liver. 2015;9(1):5-17. doi:10.5009/ gnl14118

3. Batista TP, Santos CA, Almeida GF. Perioperative chemotherapy in locally advanced gastric cancer. Arq Gastroenterol. 2013;50 (3):236-242. doi:10.1590/S0004-28032013000200042

4. Shi WJ, Gao JB. Molecular mechanisms of chemoresistance in gastric cancer. World J Gastrointest Oncol. 2016;8(9):673-681. doi:10.4251/wjgo.v8.i9.673

5. Xie Z, Klionsky DJ. Autophagosome formation: core machinery and adaptations. Nat Cell Biol. 2007;9(10):1102-1109. doi:10.1038/ncb1007-1102

6. Nawrocki ST, Wang W, Carew JS. Autophagy: new insights into its roles in cancer progression and drug resistance. Cancers. 2020;12(10):3005. doi:10.3390/cancers 12103005

7. Maiuri MC, Tasdemir E, Criollo A, et al. Control of autophagy by oncogenes and tumor suppressor genes. Cell Death Differ. 2009;16:87-93. doi:10.1038/cdd.2008.131

8. Botti J, Djavaheri-Mergny M, Pilatte Y, Codogno P. Autophagy signaling and the cogwheels of cancer. Autophagy. 2006;2:67-73. doi:10.4161/auto.2.2.2458

9. Jacob JA, Salmani JMM, Jiang Z, et al. Autophagy:an overview and its roles in cancer and obesity. Clin Chim Acta 2017;468:85-89. doi:10.1016/j.cca.2017.01.028

10. Nakatogawa H, Lane JD. Two ubiquitin-like conjugation systems that mediate membrane formation during autophagy. Essays Biochem. 2013;55:39-50. doi:10.1042/bse0550039

11. Qu B, Yao L, Ma HL, et al. Prognostic significance of autophagy-related proteins expression in resected human gastric adenocarcinoma. J Huazhong Univ Sci Technolog Med Sci. 2017;37(1):37-43. doi:10.1007/s11596-017-1691-2

12. Cao QH, Liu F, Yang ZL, et al. Prognostic value of autophagy related proteins ULK1, Beclin 1, ATG3, ATG5, ATG7, ATG9, ATG10, ATG12, LC3B and p62/SQSTM1 in gastric cancer. Am $J$ Transl Res. 2016;8(9):3831-3847.

13. Hu YF, Lei X, Zhang HY, et al. Expressions and clinical significance of autophagy-related markers Beclin1, LC3, and EGFR in human cervical squamous cell carcinoma. Onco Targets Ther. 2015;8:2243-2249. doi:10.2147/OTT.S86844

14. Zheng Y, Tu J, Wang X, et al. The therapeutic effect of melatonin on GC by inducing cell apoptosis and autophagy induced by endoplasmic reticulum stress. Onco Targets Ther. 2019;12:10187-10198. doi:10.2147/OTT.S226140

15. Du Y, Ji X. Bcl-2 down-regulation by small interfering RNA induces Beclin1-dependent autophagy in human SGC-7901 cells. Cell Biol Int. 2014;38:1155-1162. doi:10.1002/cbin.10333
16. Maiuri MC, Zalckvar E, Kimchi A, et al. Self-eating and self-killing: crosstalk between autophagy and apoptosis. Nat Rev Mol Cell Biol. 2007;8(9):741-752. doi:10.1038/nrm2239

17. Weng J, Xiao J, Mi Y, et al. PCDHGA9 acts as a tumor suppressor to induce tumor cell apoptosis and autophagy and inhibit the EMT process in human gastric cancer. Cell Death Dis. 2018;9(2). doi:10.1038/s41419-017-0189-y

18. Jung C, Ro S, Cao J, Otto N, Kim D. mTOR regulation of autophagy. FEBS Lett. 2010;584:1287-1295. doi:10.1016/j. febslet.2010.01.017

19. Mirzoeva OK, Hann B, Hom YK, et al. Autophagy suppression promotes apoptotic cell death in response to inhibition of the PI3K-mTOR pathway in pancreatic adenocarcinoma. $J$ Mol Med (Berl). 2011;89:877-889. doi:10.1007/s00109-011-0774-y

20. Wang Z-C, Huang F-Z, Xu H-B, et al. MicroRNA-137 inhibits autophagy and chemosensitizes pancreatic cancer cells by targeting ATG5. Int J Biochem Cell Biol. 2019;111:63-71. doi:10.1016/ j.biocel.2019.01.020

21. Ge J, Chen Z, Huang J, et al. Upregulation of autophagy-related gene-5 (ATG-5) is associated with chemoresistance in human gastric cancer. PLoS One. 2014;9(10):e110293. doi:10.1371/journal.pone. 0110293

22. Silberman A, Goldman O, Assayag OB, et al. Acid-induced downregulation of ASS1 contributes to the maintenance of intracellular pH in cancer. Cancer Res. 2019;79(3):518-533. doi:10.1158/0008-5472.CAN-18-1062

23. Huang $\mathrm{HY}, \mathrm{Wu}$ WR, Wang YH. ASS1 as a novel tumor suppressor gene in myxofibrosarcomas: aberrant loss via epigenetic DNA methylation confers aggressive phenotypes, negative prognostic impact, and therapeutic relevance. Clin Cancer Res. 2013;19:2861-2872. doi:10.1158/1078-0432. CCR-12-2641

24. Allen MD, Luong P, Hudson C, et al. Prognostic and therapeutic impact of argininosuccinate synthetase 1 control in bladder cancer as monitored longitudinally by PET imaging. Cancer Res. 2014;74:896-907. doi:10.1158/0008-5472.CAN-13-1702

25. Kobayashi E, Masuda M, Nakayama R, et al. Reduced argininosuccinate synthetase is a predictive biomarker for the development of pulmonary metastasis in patients with osteosarcoma. Mol Cancer Ther. 2010;9(3):535-544. doi:10.1158/1535-7163.MCT09-0774

26. Tsai CY, Chi HC, Chi LM. Argininosuccinate synthetase 1 contributes to gastric cancer invasion and progression by modulating autophagy. THE FASEB Journal. 2018;32(5):2601-2614. doi:10.1096/fj.201700094r

27. Waby JS, Bingle CD, Corfe BM. Post-translational control of sp-family transcription factors. Curr Genomics. 2008;9 (5):301-311. doi:10.2174/138920208785133244

28. Wang L, Wei D, Huang S, et al. Transcription factor Sp1 expression is a significant predictor of survival in human gastric cancer. Clin Cancer Res. 2003;9(17):6371-6380.

29. Xu XW, Pan CW, Yang XM, et al. SP1 reduces autophagic flux through activating p62 in gastric cancer cells. Mol Med Rep. 2018;17(3):4633-4638. doi:10.3892/mmr.2018.8400

30. Yates LA, Norbury CJ, Gilbert RJC. The long and short of microRNA. Cell. 2013;153(3):516-519. doi:10.1016/j. cell.2013.04.003

31. Lin S, Gregory RI. MicroRNA biogenesis pathways in cancer Nat Rev Cancer. 2015;15(6):321-333. doi:10.1038/nrc3932

32. Song JH, Meltzer SJ. MicroRNAs in pathogenesis, diagnosis, and treatment of gastroesophageal cancers. Gastroenterology. 2012;143(1):35-47. doi:10.1053/j.gastro.2012.05.003

33. Boudeau J, Baas AF, Deak M, et al. MO25alpha/beta interact with STRADalpha/beta enhancing their ability to bind, activate and localize LKB1 in the cytoplasm. EMBO J. 2003;22 (19):5102-5114. doi:10.1093/emboj/cdg490 
34. Brajenovic M, Joberty G, Kuster B, Bouwmeester T, Drewes G. Comprehensive proteomic analysis of human Par protein complexes reveals an interconnected protein network. J Biol Chem. 2004;279:12804-12811. doi:10.1074/jbc.M312171200

35. Hawley SA, Boudeau J, Reid JL, et al. Complexes between the LKB1 tumor suppressor, STRAD alpha/beta and MO25 alpha/ beta are upstream kinases in the AMP-activated protein kinase cascade. J Biol. 2003;2:28. doi:10.1186/1475-4924-2-28

36. Woods A, Johnstone SR, Dickerson K, Leiper FC, Fryer LGD. LKB1 is the upstream kinase in the AMP-activated protein kinase cascade. Curr Biol. 2003;13:2004-2008. doi:10.1016/j.cub.2003.10.031

37. Xu Z, Li Z, Wang W, Xia Y. MIR-1265 regulates cellular proliferation and apoptosis by targeting calcium binding protein 39 in gastric cancer and, thereby, impairing oncogenic autophagy. Cancer Lett. 2019;449:226-236. doi:10.1016/j.canlet.2019.02.026

38. Chen $\mathrm{S}, \mathrm{Wu}$ J, Jiao K, et al. MicroRNA-495-3p inhibits multidrug resistance by modulating autophagy through GRP78/mTOR axis in gastric cancer. Cell Death Dis. 2018;9(11). doi:10.1038/ s41419-018-0950-X

39. Yuan KT, Li BX, Yuan YJ, et al. Deregulation of microRNA-375 inhibits proliferation and migration in gastric cancer in association with autophagy-mediated AKT/mTOR signaling pathways. Technol Cancer Res Treat. 2018;17:153303381880649. doi:10.1177/1533033818806499

40. Gu Y, Fei Z, Zhu R. MiR-21 modulates cisplatin resistance of gastric cancer cells by inhibiting autophagy via the PI3K/Akt/ mTOR pathway. Anticancer Drugs. 2020;31(4):385-393. doi:10.1097/CAD.0000000000000886

41. Tian L, Zhao Z, Xie L, Zhu J. MiR-361-5p suppresses chemoresistance of gastric cancer cells by targeting FOXM1 via the PI3K/ Akt/mTOR pathway. Oncotarget. 2018;9(4):4886-4896. doi:10.18632/oncotarget.23513

42. Zhao J, Nie Y, Wang H, Lin Y. miR-181a suppresses autophagy and sensitizes gastric cancer cells to cisplatin. Gene. 2016;576 (2):828-833. doi:10.1016/j.gene.2015.11.013

43. Du X, Liu B, Luan X, et al. miR-30 decreases multidrug resistance in human gastric cancer cells by modulating cell autophagy. Exp Ther Med. 2018;15:599-605. doi:10.3892/etm.2017.5354

44. Fan H, Jiang M, Li B, et al. MicroRNA-let-7a regulates cell autophagy by targeting Rictor in gastric cancer cell lines MGC-803 and SGC-7901. Oncol Rep. 2018;39(3):1207-1214. doi:10.3892/or.2018.6194

45. Zhang $X$, Li Z, Xuan Z, et al. Novel role of miR-133a-3p in repressing gastric cancer growth and metastasis via blocking autophagy-mediated glutaminolysis. J Exp Clin Cancer Res. 2018;37(1). doi:10.1186/s13046-018-0993-y

46. Guo W, Chen Z, Chen Z, et al. Promotion of cell proliferation through inhibition of cell autophagy signalling pathway by Rab3IP is restrained by microRNA-532-3p in gastric cancer. $J$ Cancer. 2018;9(23):4363-4373. doi:10.7150/jca.27533

47. Yin X, Cao L, Kang R, et al. UV irradiation resistance-associated gene suppresses apoptosis by interfering with BAX activation. EMBO Rep. 2011;12:727-734. doi:10.1038/embor.2011.79

48. Yuan Y, Zhang Y, Han L, et al. miR-183 inhibits autophagy and apoptosis in gastric cancer cells by targeting ultraviolet radiation resistance-associated gene. Int $J$ Mol Med. 2018;42 (6):3562-3570. doi:10.3892/ijmm.2018.3871

49. Li H, He C, Wang X. MicroRNA-183 affects the development of gastric cancer by regulating autophagy via MALAT1-miR183-SIRT1 axis and PI3K/AKT/mTOR signals. Artif Cells Nanomed Biotechnol. 2019;47(1):3163-3171. doi:10.1080/ 21691401.2019.1642903

50. Xin L, Zhou LQ, Liu L, et al. METase promotes cell autophagy via promoting SNHG5 and suppressing miR-20a in gastric cancer. Int J Biol Macromol. 2019;122:1046-1052. doi:10.1016/j. ijbiomac.2018.09.051
51. Chen JF, Wu P, Xia R, et al. STAT3-induced lncRNA HAGLROS overexpression contributes to the malignant progression of gastric cancer cells via mTOR signal-mediated inhibition of autophagy. Mol Cancer. 2018;17(1). doi:10.1186/s12943-017-0756-y

52. Qu L, Ding J, Chen C, et al. Exosome-transmitted IncARSR promotes sunitinib resistance in renal cancer by acting as a competing endogenous RNA. Cancer Cell. 2016;29:653-668. doi:10.1016/j.ccell.2016.03.004

53. Fan Y, Shen B, Tan M, et al. Long non-coding RNA UCA1 increases chemoresistance of bladder cancer cells by regulating Wnt signaling. FEBS J. 2014;281:1750-1758. doi:10.1111/ febs. 12737

54. Özeş AR, Miller DF, Özeş ON, et al. NF-kB-HOTAIR axis links DNA damage response, chemoresistance and cellular senescence in ovarian cancer. Oncogene. 2016;35:5350-5361. doi:10.1038/ onc. 2016.75

55. Zheng J, Huang X, Tan W, et al. Pancreatic cancer risk variant in LINC00673 creates a miR-1231 binding site and interferes with PTPN11 degradation. Nat Genet. 2016;48:747-757. doi:10.1038/ ng.3568

56. Ma MZ, Zhang $\mathrm{Y}$, Weng $\mathrm{M}$, et al. Long non-coding RNA GCASPC, a target of miR-17-3p, negatively regulates pyruvate carboxylase-dependent cell proliferation in gallbladder cancer. Cancer Res. 2016;76:5361-5371. doi:10.1158/0008-5472.CAN15-3047

57. Wang SH, Zhang WJ, Xc W, et al. The lncRNA MALAT1 functions as a competing endogenous RNA to regulate MCL-1 expression by sponging miR-363-3p in gallbladder cancer. $J$ Cell Mol Med. 2016;20:2299-2308. doi:10.1111/jcmm.12920

58. YiRen H, YingCong Y, Sunwu Y, et al. Long noncoding RNA MALAT1 regulates autophagy associated chemoresistance via miR-23b-3p sequestration in gastric cancer. Mol Cancer. 2017;16(1):1-12. doi:10.1186/s12943-017-0743-3

59. Zhu L, Zhu Y, Han S, et al. Impaired autophagic degradation of IncRNA ARHGAP5-AS1 promotes chemoresistance in gastric cancer. Cell Death Dis. 2019;10(6). doi:10.1038/s41419-019$1585-2$

60. Zhou J, Jiang YY, Chen H, et al. Tanshinone I attenuates the malignant biological properties of ovarian cancer by inducing apoptosis and autophagy via the inactivation of PI3K/AKT/ mTOR pathway. Cell Prolif. 2020;53(2):e12739. doi:10.1111/ cpr.12739

61. Wang J, Liang D, Zhang XP, et al. Novel PI3K/Akt/mTOR signaling inhibitor, W922, prevents colorectal cancer growth via the regulation of autophagy. Int J Oncol. 2021;58(1):70-82. doi:10.3892/ijo.2020.5151

62. Zheng W, Wu C, Wu X, et al. Genetic variants of autophagy-related genes in the PI3K/Akt/mTOR pathway and risk of gastric cancer in the Chinese population. Gene. 2021;769:145190. doi:10.1016/j.gene.2020.145190

63. Murakami D, Tsujitani S, Osaki T, et al. Expression of phosphorylated Akt(pAkt) in gastric carcinoma predicts prognosis and efficacy of chemotherapy. Gastric Cancer. 2007;10(1):45-51. doi:10.1007/s10120-006-0410-7

64. Yap T, Garrett M, Walton M, et al. Targeting the PI3K-AKTmTOR pathway: progress, pitfalls, and promises. Curr Opin Pharmacol. 2008;8(4):393-412. doi:10.1016/j.coph.2008.08.004

65. Raha S, Yumnam S, Hong GE, et al. Naringin induces autophagy-mediated growth inhibition by down-regulating the $\mathrm{PI} 3 \mathrm{~K} / \mathrm{Akt} / \mathrm{mTOR}$ cascade via activation of MAPK pathways in AGS cancer cells. Int $J$ Oncol. 2015;47(3):1061-1069. doi:10.3892/ijo.2015.3095

66. Guo F, Jiao D, Sui GQ, et al. Anticancer effect of YWHAZ silencing via inducing apoptosis and autophagy in gastric cancer cells. Neoplasma. 2018;65:693-700. doi:10.4149/neo_2018_170 922N603 
67. Hu XL, Zhu YJ, Hu CH, et al. Ghrelin affects gastric cancer progression by activating AMPK signaling pathway. Biochem Genet;2021:1-16. doi:10.1007/s10528-020-10010-1

68. Faubert B, Vincent EE, Poffenberger MC, et al. The AMP-activated protein kinase (AMPK) and cancer: many faces of a metabolic regulator. Cancer Lett. 2015;356:165-170. doi:10.1016/j.canlet.2014.01.018

69. Yu Z, Suosi L, Qin F, et al. Perilaldehyde activates AMP-activated protein kinase to suppress the growth of gastric cancer via induction of autophagy. J Cell Biochem. 2019;120(2):1716-1725.

70. Pei G, Luo M, Ni X, et al. Autophagy facilitates metadherin-induced chemotherapy resistance through the AMPK/ATG5 pathway in gastric cancer. Cell Physiol Biochem. 2018;46(2):847-859. doi:10.1159/000488742

71. Piscione M, Mazzone M, Di Marcantonio MC, et al. Eradication of Helicobacter pylori and gastric cancer: a controversial relationship. Front Microbiol. 2021;12. doi:10.3389/ fmicb.2021.630852

72. Díaz P, Valenzuela Valderrama M, Bravo J, et al. Helicobacter pylori and gastric cancer: adaptive cellular mechanisms involved in disease progression. Front Microbiol. 2018;9:5. doi:10.3389/ fmicb.2018.00005

73. Yahiro K, Satoh M, Nakano M, et al. Low-density lipoprotein receptor-related protein-1 (LRP1) mediates autophagy and apoptosis caused by Helicobacter pylori VacA. J Biol Chem. 2012;287 (37):31104-31115. doi:10.1074/jbc.M112.387498

74. Wang MY, Chen C, Gao XZ, et al. Distribution of Helicobacter pylori virulence markers in patients with gastroduodenal diseases in a region at high risk of gastric cancer. Microb Pathog. 2013;59:13-18. doi:10.1016/j.micpath.2013.04.001

75. Paik JY, Lee HG, Piao JY, et al. Helicobacter pylori infection promotes autophagy through Nrf2-mediated heme oxygenase upregulation in human gastric cancer cells. Biochem Pharmacol. 2019;162:89-97. doi:10.1016/j.bcp.2019.02.003

76. Liu X, Zheng Q, Yu Q, et al. Apatinib regulates the growth of gastric cancer cells by modulating apoptosis and autophagy. Naunyn Schmiedebergs Arch Pharmacol. 2020. doi:10.1007/ s00210-020-02018-6

77. Hu S, Yin J, Yan S, et al. Chaetocochin J, an epipolythiodioxopiperazine alkaloid, induces apoptosis and autophagy in colorectal cancer via AMPK and PI3K/AKT/mTOR pathways. Bioorg Chem. 2021;109:104693. doi:10.1016/j.bioorg.2021.104693

78. Liu H, Song J, Zhou Y, et al. Methylxanthine derivatives promote autophagy in gastric cancer cells targeting PTEN. Anticancer Drugs. 2019;30(4):347-355. doi:10.1097/CAD.0000000000000 724

79. Okada T, Yoshida H, Akazawa R, et al. Distinct roles of activating transcription factor 6 (ATF6) and double-stranded RNA-activated protein kinase-like endoplasmic reticulum kinase(PERK) in transcription during the mammalian unfolded protein response. Biochem J. 2002;366:585-594. doi:10.1042/ bj20020391

80. Peng X, Tu Y, Fu S, et al. 14-Deoxycoleon U-induced endoplasmic reticulum stress-mediated apoptosis, autophagy, and cell cycle arrest in lung adenocarcinoma. Onco Targets Ther 2019;12:5955-5965. doi:10.2147/OTT.S211933

81. Bai XY, Liu YG, Song W, et al. Anticancer activity of tetrandrine by inducing pro-death apoptosis and autophagy in human gastric cancer cells. J Pharm Pharmacol. 2018;70(8):1048-1058. doi:10.1111/jphp. 12935

82. Klionsky DJ. Autophagy: from phenomenology to molecular understanding in less than a decade. Nat Rev Mol Cell Biol. 2007;8:931-937. doi:10.1038/nrm2245

83. Kumar A, Singh UK, Chaudhary A. Targeting autophagy to overcome drug resistance in cancer therapy. Future Med Chem. 2015;7:1535-1542. doi:10.4155/fmc. 15.88
84. Belounis A, Nyalendo C, Le Gall R, et al. Autophagy is associated with chemoresistance in neuroblastoma. BMC Cancer. 2016;16(1). doi:10.1186/s12885-016-2906-9

85. Wu J, Yu J, Wang J, et al. Astragalus polysaccharide enhanced antitumor effects of Apatinib in gastric cancer AGS cells by inhibiting AKT signalling pathway. Biomed Pharmacother. 2018;100:176-183. doi:10.1016/j.biopha.2018.01.140

86. Xiong X, Lu B, Tian Q, et al. Inhibition of autophagy enhances cinobufagin-induced apoptosis in gastric cancer. Oncol Rep. 2019;41(1):492-500. doi:10.3892/or.2018.6837

87. Klionsky DJ, Abdelmohsen K, Abe A, et al. Guidelines for the use and interpretation of assays for monitoring autophagy (3rd edition). Autophagy. 2016;12:1-222.

88. Zhang BC, Zhang CW, Wang C, et al. Luteolin attenuates foam cell formation and apoptosis in Ox-LDL-stimulated macrophages by enhancing autophagy. J Am Coll Car-Diol. 2016;39:2065-2076.

89. Schonewolf CA, Mehta M, Schiff D, et al. Autophagy inhibition by chloroquine sensitizes HT-29 colorectal cancer cells to concurrent chemoradiation. World J Gastrointest Oncol. 2014;6 (3):74-82. doi:10.4251/wjgo.v6.i3.74

90. Mauthe M, Orhon I, Rocchi C, et al. Chloroquine inhibits autophagic flux by decreasing autophagosome-lysosome fusion. Autophagy. 2018;14(8):1435-1455. doi:10.1080/15548627.2018.1474314

91. Li LQ, Pan D, Zhang SW, et al. Autophagy regulates chemoresistance of gastric cancer stem cells via the Notch signaling pathway. Eur Rev Med Pharmacol Sci. 2018;22(11):3402-3407.

92. Li S, Li J, Shen C, et al. tert-Butylhydroquinone (tBHQ) protects hepatocytes against lipotoxicity via inducing autophagy independently of Nrf2 activation. Biochim Biophys Acta. 2014;1841 (1):22-33. doi:10.1016/j.bbalip.2013.09.004

93. Wang K, Du B, Xu B, et al. JMJD6-STAT3Y705ph axis promotes autophagy in osteosarcoma cancer cells by regulating ATG. BioFactors. 2020;46:839-848. doi:10.1002/biof.1614

94. Yousefi S, Perozzo R, Schmid I, et al. Calpain-mediated cleavage of Atg5 switches autophagy to apoptosis. Nat Cell Biol. 2006;8:1124-1132. doi:10.1038/ncb1482

95. Le CP, Nowell CJ, Kim-Fuchs C, et al. Chronic stress in mice remodels lymph vasculature to promote tumour cell dissemination. Nat Commun. 2016;7:10634. doi:10.1038/ ncomms 10634

96. Krizanova O, Babula P, Pacak K. Stress, catecholaminergic system and cancer. Stress. 2016;19:419-428. doi:10.1080/ 10253890.2016 .1203415

97. Zhi X, Li B, Li Z, et al. Adrenergic modulation of AMPK-dependent autophagy by chronic stress enhances cell proliferation and survival in gastric cancer. Int J Oncol. 2019;54 (5):1625-1638. doi:10.3892/ijo.2019.4753

98. Xu W, Shi Q, Qian X. Rab5a suppresses autophagy to promote drug resistance in cancer cells. Am J Transl Res. 2018;10 (4):1229-1236

99. Kim J, Kundu M, Viollet B, Guan KL. AMPK and mTOR regulate autophagy through direct phosphorylation of Ulk1. Nat Cell Biol. 2011;13:132-141. doi:10.1038/ncb2152

100. Hardie DG. AMP-activated protein kinase: an energy sensor that regulates all aspects of cell function. Genes Dev. 2011;25:1895-1908. doi:10.1101/gad.17420111

101. Kim TW, Lee SY, Kim M, et al. DSGOST regulates resistance via activation of autophagy in gastric cancer. Cell Death Dis. 2018;9 (6):1-13. doi:10.1038/s41419-018-0658-y

102. Gurpinar E, Grizzle WE, Piazza GA. NSAIDs inhibit tumorigenesis, but how? Clin. Cancer Res. 2014;20:1104-1113.

103. Vallecillo-Hernández J, Barrachina MD, Ortiz-Masiá D, et al. Indomethacin disrupts autophagic flux by inducing lysosomal dysfunction in gastric cancer cells and increases their sensitivity to cytotoxic drugs. Sci Rep. 2018;8(1):1-10. doi:10.1038/s41598$018-21455-1$ 
104. Chen J, Shao R, Li F, et al. PI3K/Akt/mTOR pathway dual inhibitor BEZ235 suppresses the stemness of colon cancer stem cells Clin Exp Pharmacol Physiol. 2015;42(12):1317-1326. doi:10.1111/1440-1681.12493

105. Yin AH, Miraglia S, Zanjani ED, et al. AC133, a novel marker for human hematopoietic stem and progenitor cells. Blood. 1997;90 (12):5002-5012. doi:10.1182/blood.V90.12.5002

106. Lu R, Zhao G, Yang Y, et al. Inhibition of CD133 overcomes cisplatin resistance through inhibiting PI3K/AKT/mTOR signaling pathway and autophagy in CD133-positive gastric cancer cells. Technol Cancer Res Treat. 2019;18:153303381986431. doi:10.1177/1533033819864311

107. Lim H, Son KH, Chang HW, Bae K, Kang SS, Kim HP. Antiinflammatory activity of pectolinarigenin and pectolinarin isolated from Cirsium chanroenicum. Biol Pharm Bull. 2008;31:2063-2067. doi:10.1248/bpb.31.2063

108. Zhang T, Li S, Li J, et al. Natural product pectolinarigenin inhibits osteosarcoma growth and metastasis via SHP-1-mediated STAT3 signaling inhibition. Cell Death Dis. 2016;7:e2421-e2421. doi:10.1038/cddis.2016.305

109. Lee HJ, Venkatarame Gowda Saralamma V, Kim SM, et al. Pectolinarigenin induced cell cycle arrest, autophagy, and apoptosis in gastric cancer cell via $\mathrm{PI} 3 \mathrm{~K} / \mathrm{AKT} / \mathrm{mTOR}$ signaling pathway. Nutrients. 2018;10(8):1043. doi:10.3390/nu10081043

110. Bajpai M, Pande A, Tewari SK, Prakash D. Phenolic contents and anti-oxidant activity of some food and medicinal plants. Int $J$ Food Sci Nutr. 2005;56:287-291. doi:10.1080/ 09637480500146606

111. Kim TW, Lee SY, Kim M, et al. Kaempferol induces autophagic cell death via IRE1-JNK-CHOP pathway and inhibition of G9a in gastric cancer cells. Cell Death Dis. 2018;9(9):1-14. doi:10.1038/ s41419-018-0930-1

112. Zhang F, Ma C. Kaempferol suppresses human gastric cancer SNU-216 cell proliferation, promotes cell autophagy, but has no influence on cell apoptosis. Braz J Med Biol Res. 2019;52(2). doi:10.1590/1414-431x20187843

113. Kumar A, Sirohi VK, Anum F, et al. Enhanced apoptosis, survivin down-regulation and assisted immunochemotherapy by curcumin loaded amphiphilic mixed micelles for subjugating endometrial cancer. Nanomedicine. 2017;13(6):1953-1963. doi:10.1016/j.nano.2017.04.014

114. Pandey A, Vishnoi K, Mahata S, et al. Berberine and curcumin target survivin and STAT3 in gastric cancer cells and synergize actions of standard chemotherapeutic 5-Fluorouracil. Nutr Cancer. 2015;67 (8):1293-1304. doi:10.1080/01635581.2015.1085581

115. Zou P, Xia Y, Chen T, et al. Selective killing of gastric cancer cells by a small molecule targeting ROS-mediated ER stress activation. Mol Carcinog. 2016;55(6):1073-1086. doi:10.1002/ mc. 22351

116. Yu LL, Wu JG, Dai N, et al. Curcumin reverses chemoresistance of human gastric cancer cells by downregulating the NF- $\mathrm{KB}$ transcription factor. Oncol Rep. 2011;26(5):1197-1203. doi:10.3892/or.2011.1410

117. Bengmark S. Curcumin, an atoxic antioxidant and natural NF-kB, cyclooxygenase-2, lipooxygenase, and inducible nitric oxide synthase inhibitor: a shield against acute and chronic diseases. $J$ Parenter Enteral Nutr. 2006;30(1):45-51. doi:10.1177/ 014860710603000145

118. Fu H, Wang C, Yang D, et al. Curcumin regulates proliferation, autophagy, and apoptosis in gastric cancer cells by affecting PI3K and P53 signaling. $J$ Cell Physiol. 2018;233(6):4634. doi:10.1002/jep.26190

119. Li C, Wang MH. Potential biological activities of magnoflorine: a compound from Aristolochia debilis Sieb. et Zucc. Korean J Plant Resour. 2014;27:223-228. doi:10.7732/kjpr.2014.27. 3.223
120. Zhao Z, Wang J, Tang J, et al. JNK-and Akt-mediated Puma expression in the apoptosis of cisplatin resistant ovarian cancer cells. Biochem J. 2012;444:291-301. doi:10.1042/ BJ20111855

121. Sun XL, Zhang XW, Hj Z, et al. Magnoflorine inhibits human gastric cancer progression by inducing autophagy, apoptosis and cell cycle arrest by JNK activation regulated by ROS. Biomed Pharmacother. 2020;125:109118. doi:10.1016/j.biopha.2019.10 9118

122. Acuna-Castroviejo D, Escames G, Venegas C, et al. Extrapineal melatonin: sources, regulation, and potential functions. $\mathrm{Cel} \mathrm{Mol}$ Life Sci. 2014;71(16):2997-3025.

123. Song J, Ma SJ, Luo JH, et al. Melatonin induces the apoptosis and inhibits the proliferation of human gastric cancer cells via blockade of the AKT/MDM2 pathway. Oncol Rep. 2018;39 (4):1975-1983. doi:10.3892/or.2018.6282

124. Ron D, Walter P. Signal integration in the endoplasmic reticulum unfolded protein response. Nat Rev Mol Cell Biol. 2007;8 (7):519-529. doi:10.1038/nrm2199

125. Ogata M, Hino SI, Saito A, et al. Autophagy is activated for cell survival after endoplasmic reticulum stress. Mol Cell Biol. 2006;26(24):9220-9231. doi:10.1128/MCB.01453-06

126. Sano R, Reed JC. ER stress-induced cell death mechanisms. Biochim Biophys Acta. 2013;1833(12):3460-3470. doi:10.1016/ j.bbamcr.2013.06.028

127. Lee H, Lee S, Jeong D, Kim SJ. Ginsenoside Rh2 epigenetically regulates cell-mediated immune pathway to inhibit proliferation of MCF-7 breast cancer cells. J Ginseng Res. 2018;42 (4):455-462. doi:10.1016/j.jgr.2017.05.003

128. Sun D, Lu M, Wang S, Fu L. Ginsenoside Rg3 inhibits gastric cancer cell proliferation through $\mathrm{Ca}^{2+} / \mathrm{CaM}$ kinase downregulation and NF-kB inactivation. Chin J Cancer Biother. 2015;22 (2):225-229.

129. Mao Q, Zhang PH, Wang Q, Li SL. Ginsenoside F2 induces apoptosis in humor gastric carcinoma cells through reactive oxygen species-mitochondria pathway and modulation of ASK-1/ JNK signaling cascade in vitro and in vivo. Phytomedicine. 2014;21(4):515-522. doi:10.1016/j.phymed.2013.10.013

130. Liu Y, Fan D. Ginsenoside Rg5 induces G2/M phase arrest, apoptosis and autophagy via regulating ROS-mediated MAPK pathways against human gastric cancer. Biochem Pharmacol. 2019;168:285-304. doi:10.1016/j.bcp.2019.07.008

131. Kakuyama A, Sadzuka Y. Effect of methylxanthine derivatives on doxorubicin transport and antitumor activity. Curr Drug Metab. 2001;2:379-395. doi:10.2174/1389200013338270

132. Liu H, Zhou Y, Tang L. Caffeine induces sustained apoptosis of human gastric cancer cells by activating the caspase $9 /$ caspase 3 signalling pathway. Mol Med Rep. 2017;16:2445-2454. doi: $10.3892 / \mathrm{mmr} .2017 .6894$

133. Mukhopadhyay S, Panda PK, Sinha N, Das DN, Bhutia SK. Autophagy and apoptosis: where do they meet? Apoptosis. 2014;19:555-566. doi:10.1007/s10495-014-0967-2

134. Liu W, Zhang B, Chen G, et al. Targeting miR-21 with sophocarpine inhibits tumor progression and reverses epithelial-mesenchymal transition in head and neck cancer. Mol Ther. 2017;25(9):2129-2139. doi:10.1016/j.ymthe.2017.05.008

135. Huang Y, Chen X, Guo G, et al. Sophocarpine inhibits the growth of gastric cancer cells via autophagy and apoptosis. Front Biosci. 2019;24:616-627.

136. Lim SC, Han SI. Ursodeoxycholic acid effectively kills drug-resistant gastric cancer cells through induction of autophagic death. Oncol Rep. 2015;34:1261-1268. doi:10.3892/or.2015.4076

137. Xu MY, Lee DH, Joo EJ, Son KH, Kim YS. Akebia saponin PA induces autophagic and apoptotic cell death in AGS human gastric cancer cells. Food Chem Toxicol. 2013;59:703-708. doi:10.1016/j.fct.2013.06.059 


\section{Publish your work in this journal}

The International Journal of General Medicine is an international, peer-reviewed open-access journal that focuses on general and internal medicine, pathogenesis, epidemiology, diagnosis, monitoring and treatment protocols. The journal is characterized by the rapid reporting of reviews, original research and clinical studies across all disease areas. The manuscript management system is completely online and includes a very quick and fair peer-review system, which is all easy to use. Visit http://www.dovepress.com/ testimonials.php to read real quotes from published authors.

Submit your manuscript here: https://www.dovepress.com/international-journal-of-general-medicine-journal 